\section{Donepezil Versus Rivastigmine in Patients with Alzheimer's Disease: Attention and Working Memory}

\section{Paul S Foster ${ }^{1,3 *}$, Valeria Drago ${ }^{3,4}$, Katelyn M Roosa ${ }^{1}$, Ransom W Campbell ${ }^{5}$, John C Witt ${ }^{2}$ and Kenneth M Heilman ${ }^{3}$}

${ }^{1}$ Department of Psychology, Middle Tennessee State University, Greenland Drive, Murfreesboro, TN, USA

${ }^{2}$ Murfreesboro Medical Clinic, Garrison Drive, Murfreesboro, TN, USA

${ }^{3}$ McKnight Brain Institute, University of Florida, S Newell Drive, Gainesville, FL, USA

"UOC Neurologia, ASP Siracusa, Ospedale "Muscatello" Augusta, Siracusa, Italy

${ }^{5}$ Department of Psychology, Virginia Tech, Blacksburg, VA, USA

\section{Introduction}

A number of studies have demonstrated the negative impact of cholinergic disruption on attention. For example, research has indicated that the anticholinergic (antimuscarinic) agent scopolamine impairs working memory and attention [1]. Other researchers have reported a broadening of spatial attention following administration of scopolamine, indicating that acetylcholine may have an important role in narrowing the scale of attentional focus [2]. Broks and colleagues reported that scopolamine impairs sustained attention, disrupting the ability of individuals to impose voluntary attention over extended periods of time [3] and the ability to sustain attention (vigilance) is critical for working memory tests. Further, whereas the administration of scopolamine reduces the maintenance of working memory and selective attention, cholinergic enhancement through the administration of physostigmine improves the maintenance of selective attention and working memory [4].

Reduced levels of Choline Acetyltransferase (ChAT) have been reported in patients with $\mathrm{AD}$ and a relationship exists between the reduction of ChAT and the density of neuritic plaques and neurofibrillary tangles [5]. Reduced levels Acetylcholinesterase (AChE) and increased levels of Butyrylcholinesterase (BChE) in the hippocampus and temporal cortex of patients with $\mathrm{AD}$ have also been reported [6]. Although the cortical laminar distribution of AChE and $\mathrm{BChE}$ varies [7], the neuritic plaques and neurofibrillary tangles have been found to contain both AChE and BChE [8]. Functional neuroimaging studies have reported in vivo degeneration of the cholinergic system in the frontal, parietal, and temporal cortex of patients with $\mathrm{AD}$, as well as in the hippocampus [9]

The degeneration of the cholinergic system in $\mathrm{AD}$ has profound effects on working memory and attention functioning in these patients [10]. Patients with AD have reduced or impaired selective attention [11,12] and divided attention [13]. The performance of $\mathrm{AD}$ patients on tests of attention is correlated with changes in $\mathrm{AChE}$ activity and 11C-nicotine binding [14]. Finally, cortical AChE activity in the dorsolateral prefrontal cortex is significantly related to performance on measures of working memory and attention in $\mathrm{AD}$ patients, but not to episodic memory functioning [15].

Acetylcholinesterase Inhibitors (AChEIs) have emerged as the primary treatment for the memory problems associated with Alzheimer's disease. However, given the relationship between attention, working memory, and cholinergic activity in patients with Alzheimer's disease, AChEI medications may also help to increase attentional functioning and working memory in these patients. Attention functioning may be "bottom-up" such as when a person calls your name, or "top-down" intentional/selective such as when you are listening to someone speak to you during a party or during a working memory task. Some have proposed that in the regions of the brain important for attentional functioning, including the prefrontal cortex, Acetylcholine (ACh) may have a vital role in the top-down control of attention [16]. Cholinergic medications such as donepezil and rivastigmine are associated with significant reduction in AChE activity in the cerebral cortex, with greater AChE inhibition in the 
frontal cortex [17], suggesting that these medications may have a strong influence on intentional selective attention and working memory.

Improved attention functioning following treatment with donepezil has been reported by many investigators [18], including sustained attention [19], and a measure of selective visual attention [20]. Bohnen et al., [21] reported improved performance in patients with $\mathrm{AD}$ following treatment with donepezil and that the degree of inhibition of cortical AChE was related to performance on tests of attention. In regard to working memory, performance on a digit span task has been reported to improve following administration of donepezil [22]. Further, a significant relationship has been reported between donepezil-binding AChE density in the brain and performance on the Digit Symbol subtest of the Wechsler Adult Intelligence Scale [23].

Rivastigmine is another commonly prescribed AChEI and studies have indicated that this medication is also associated with improvement in attention [24-26]. Whereas patients with $\mathrm{AD}$ who are treated with rivastigmine have shown stability on the Digit Symbol subtest and Part A of the Trail Making Test (TMT-A), those who were untreated evidenced decline on these measures [27]. Additionally, this investigation also reported increases in regional cerebral blood flow in the frontal and temporal regions following treatment with rivastigmine. Darreh-Shori et al., [28] reported significant correlations between the reduction of $\mathrm{AChE}$ activity following treatment with rivastigmine and performance on tests of attention.

There have been numerous studies conducted comparing the effects of donepezil and rivastigmine on general cognitive functioning. Reviews have reported comparable effects of these medications on measures such as the Mini Mental State Examination (MMSE) and the AD Assessment Scale - Cognitive [29,30]. However, Caffarra et al., [31] reported greater stability in MMSE scores across time for $\mathrm{AD}$ patients treated with rivastigmine than for those treated with donepezil. Others have reported improvements on measures of cognitive functioning over time with rivastigmine but only stability with donepezil [32]. Scharre and colleagues [33] found that patients with $\mathrm{AD}$ treated with rivastigmine had a lower rate of prescription of antipsychotic medication that those treated with donepezil. Although a number of studies have compared donepezil and rivastigmine on measures of general cognitive functioning, there have been no investigations reported that have directly compared the effect of these medications on measures of working memory and attention. Hence, the purpose of the present investigation was to determine whether differential effects on measures of working memory and attention exist for $\mathrm{AD}$ patients taking donepezil versus rivastigmine.

A primary difference between donepezil and rivastigmine is that whereas donepezil is specific for inhibiting $\mathrm{AChE}$, rivastigmine is known to inhibit both AChE and BChE. Given that AChE and BChE are both known to hydrolyze ACh [34] and the aforementioned important role of the cholinergic system in attention, this might suggest an advantage for rivastigmine in the treatment of the attentional problems associated with $\mathrm{AD}$. Some investigators have reported that whereas $\mathrm{BChE}$ inhibition in cerebral spinal fluid is related to performance on a measure of attention, AChE inhibition was not [35]. Hence, we predicted that rivastigmine would be associated with greater benefits on indices of attention and working memory, as compared to donepezil.

\section{Methods}

\section{Participants}

The study was approved by the Institutional Review Board of Middle Tennessee State University. The sample consisted of 12 patients with $\mathrm{AD}$ who were taking donepezil, 12 patients with $\mathrm{AD}$ who were taking rivastigmine, and 12 patients with $\mathrm{AD}$ who were not taking any AChEI. Regarding the dosages of the medications, six patients were taking $5 \mathrm{mg}$ and six patients were taking $10 \mathrm{mg}$ of donepezil. A total of seven patients were taking $4.6 \mathrm{mg}$ of rivastigmine, three were taking $9.5 \mathrm{mg}$, and one each were taking $3 \mathrm{mg}$ and $6 \mathrm{mg}$ dosages of rivastigmine. The diagnosis of $\mathrm{AD}$ met the NINDS-ADRDA criteria for probable AD and DSM-IVTR criteria. The patient sample was drawn from patients who were referred for a neuropsychological evaluation for memory problems to the Memory Disorder Clinic at the Murfreesboro Medical Clinic. We should note that, at the time of the evaluation, there were some patients who also seemed to have a vascular component and were thereby diagnosed with $\mathrm{AD}$ as well as Vascular Dementia (VD). However, patient diagnosis was matched across the three groups such that there were equal numbers of patients who also had VD $(n=3)$. Also, some of our patients were taking Namenda at the time of the evaluation. As before, the number of patients also taking Namenda was matched across groups such that there were equal numbers ( $n=2$ per group). All patients who were also taking Namenda were diagnosed with only AD. The total sample of 36 patients included 7 men and 29 women with an age range of 60 to 87 years $(\mathrm{M}=77.47, \mathrm{SD}=7.10)$. The average education level for the entire sample was 10.75 years $(\mathrm{SD}=2.88)$ and the average MMSE score was 20.00 ( $\mathrm{SD}=3.14$ ). Consult table 1 for the demographic information associated with each group.

\begin{tabular}{|c|c|c|c|}
\hline & Donepezil & Rivastigmine & No Treatment \\
\hline Sex & 9 women / 3 men & 10 women / 2 men & 10 women / 2 men \\
\hline Age & $73.92(8.89)$ & $81.58(3.63)$ & $76.92(5.98)$ \\
\hline Education & $11.67(2.84)$ & $10.92(2.71)$ & $9.67(2.96)$ \\
\hline GDS & $9.55(5.41)$ & $7.40(4.95)$ & $9.18(5.23)$ \\
\hline MMSE & $20.42(3.78)$ & $19.92(3.53)$ & $19.67(2.06)$ \\
\hline
\end{tabular}

Table 1: Basic demographic information for the three groups.

Note: Means are reported with standard deviations in parentheses. GDS refers to the Geriatric Depression Scale. MMSE refers to the Mini Mental State Examination.

\section{Apparatus}

\section{Dementia rating scale - attention}

The attention subtest of the Dementia Rating Scale revised (DRSatt) consists of a variety of tasks that are purported to measure basic attentional functioning. The tasks include a digit span forward task requiring the patient to repeat a series of digits that are aurally presented and a digit span backward task that requires the patient to repeat the digits in the reverse order as presented. Other tasks include a motor command task, a task requiring the patient to consecutively read a list of five words, and another task requiring the patient to follow the examiner in pointing to a series of four graphic designs. We should note that the DRSatt data was not available for one patient. The dependent variable of interest in this study was the age corrected percentile score.

\section{Digit span}

The Digit Span (DS) subtest of the Wechsler Memory Scale-III (WMS-III) is a measure of attention and working memory that 
requires the patient to repeat strings of digits of increasing length that are spoken by the examiner. The DS subtest consists of two conditions, one in which the patient repeats the string of digits as presented by the examiner and another requiring the subject to repeat the numbers in the reversed order. The dependent variables of interest in this study included of the age corrected percentile score from the total subtest (DSt) and the age corrected percentile score from the forward condition (DSf).

\section{Digit symbol}

The Digit Symbol (DSymb) subtest of the Wechsler Adult Intelligence Scale-III (WAIS-III) is a measure of attention and processing speed. The test pairs nine digits (1 through 9) with nine different symbols in a key code at the top of the page. The remainder of the page consists of a series of boxes with the top portion containing the digits in a pseudorandom fashion and the boom portion being empty. The task requires the patient to write the symbol for each digit in the empty portion of the box. The dependent variable of interest in our study was the age corrected percentile score from the total number of boxes corrected completed. We should note that this data was not available for three of our patients.

\section{Geriatric depression scale}

The Geriatric Depression Scale (GDS) is a 30 item self-report questionnaire designed for use with older populations. Participants are asked to respond either "yes" or "no" to each item, with a range of possible scores from 0 to 30 .

\section{Mini mental status exam}

The Mini Mental State Examination (MMSE) is a screening test used to assess general cognitive functioning. Areas of functioning assessed include orientation, registration, attention, recall, working memory, language, and construction or drawing ability. The range of scores possible is from 0 to 30 .

\section{Procedure}

The study was approved by the Institutional Review Board of the primary author's institution in the United States and all participants provided written informed consent. The DRSatt, DS, DSymb, GDS, and MMSE were all administered according to standard procedures, as described previously. The tests were administered in a pseudorandom fashion, although the MMSE was always administered first.

\section{Result}

Initial analyses were conducted to determine group equivalence in terms of age, education, GDS, and MMSE. The results of separate three-way ANOVAs performed on each of these variables indicated no significant differences between the three groups in their education, GDS, or MMSE score. However, a significant difference was noted for age between the groups, $\mathrm{F}(2,33) 4.20, \mathrm{p}=.024$. Subsequent analyses indicated that the rivastigmine group was significantly older than the donepezil group, $\mathrm{F}(1,22)=7.65, \mathrm{p}=.011$, and also significantly older than the no treatment control group, $F(1,22)=5.35, p=.031$. Please consult the table 1 for means and standard deviations for all demographic variables.

Given the significant difference in age, all subsequent analyses were conducted using this variable as a covariate in a series of ANCOVAs to control for this potentially confounding variable. A three-way between groups ANCOVA using the DRSatt data indicated a significant difference between the groups, $\mathrm{F}(2,31)=3.48$, $\mathrm{p}=.043, \mathrm{R}^{2}=.231$. Subsequent comparisons indicated that the donepezil group performed significant better than the no treatment control group, $\mathrm{F}(1,21)=7.69, \mathrm{p}=.011$. No significant differences were noted between the donepezil group and the rivastigmine group, $\mathrm{F}(1,20)=3.97, \mathrm{p}=.06$, or between the rivastigmine and the no treatment control groups, $\mathrm{F}(1,20)=.001, \mathrm{p}=.984$.

The data from the Digit Span test were then analyzed. Regarding the DSt data, a three-way between groups ANCOVA indicated a significant difference between the groups, $\mathrm{F}(2,32)=10.79, \mathrm{p}=.0003$, $\mathrm{R}^{2}=.455$. Subsequent comparisons indicated a significant difference between the donepezil group and both the rivastigmine group, $\mathrm{F}(1,21)=17.70, \mathrm{p}=.0004$, and the no treatment control group, $\mathrm{F}(1,21)=12.95, \mathrm{p}=.002$. No significant difference was noted between the rivastigmine and the no treatment control groups, $\mathrm{F}(1,21)=.004, \mathrm{p}=.951$. Similarly, the result of a three-way between subjects ANCOVA on the DSf data indicated a significant difference between the groups, $\mathrm{F}(2,32)=9.89, \mathrm{p}=.0005, \mathrm{R}^{2}=.407$. Subsequent comparisons indicated a significant difference between the donepezil group and both the rivastigmine group, $\mathrm{F}(1,21)=13.46, \mathrm{p}=.001$, and the no treatment control group, $\mathrm{F}(1,21)=12.76, \mathrm{p}=.002$. No significant difference was noted between the rivastigmine and no treatment control groups, $\mathrm{F}(1,21)=.64, \mathrm{p}=.432$.

Finally, the results of a three-way between subjects ANCOVA on the DSymb data indicated a significant difference between the groups, $\mathrm{F}(2,29)=5.04, \mathrm{p}=.013, \mathrm{R}^{2}=.406$. Subsequent comparisons indicated a significant difference between the donepezil group and the rivastigmine group, $\mathrm{F}(1,19)=5.81, \mathrm{p}=.026$, and the no treatment control group, $\mathrm{F}(1,20)=10.45, \mathrm{p}=.004$. No significant difference was found between the rivastigmine group and the no treatment control group, $\mathrm{F}(1,18)=.10, \mathrm{p}=.759$. Please consult table 2 for the means and standard deviations for all dependent variables of interest and the figure 1 for a graphical representation.

\begin{tabular}{|c|c|c|c|}
\hline & Donepezil & Rivastigmine & No Treatment \\
\hline DRSatt & $61.50(26.08)$ & $48.82(25.31)$ & $45.83(15.46)$ \\
\hline DSt & $40.00(30.33)$ & $20.25(15.33)$ & $17.50(12.82)$ \\
\hline DSf & $43.58(24.33)$ & $23.08(18.57)$ & $22.75(9.37)$ \\
\hline DSsymb & $25.92(28.44)$ & $18.00(21.65)$ & $10.36(8.18)$ \\
\hline
\end{tabular}

Table 2: Means and standard deviations for each dependent variable of interest.

Note: Means and standard deviations are reported in parentheses.

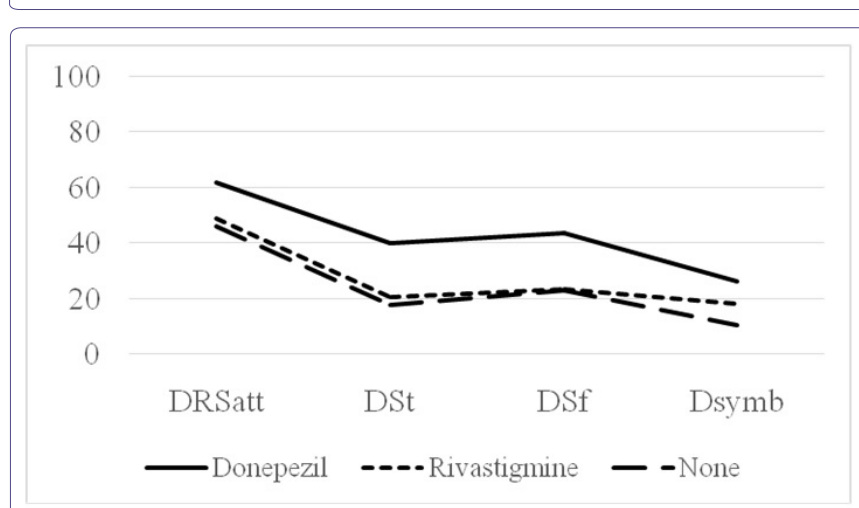

Figure 1: The effects of donepezil and rivastigmine on attention and working memory.

Note: Means and standard deviations are reported in parentheses. 


\section{Discussion}

Given the role of rivastigmine in inhibiting both AChE and $\mathrm{BChE}$ we thought that this medication might have an advantage over donepezil in treating the attention or working memory deficits often seen in patients with Alzheimer's disease, particularly since research has indicated that $\mathrm{BChE}$ inhibition is related to performance on a test of attention but AChE inhibition is not [35]. However, our data consistently indicated the opposite. The donepezil treated group evidenced significantly better performance on measures of working memory and attention when compared to the no treatment control group and also performed significantly better than the rivastigmine group on three of our four measures. There was a significant difference in age between our groups, which could have affected the findings. However, we controlled for these age differences by including age as a covariate in all analyses. Hence, the analyses and subsequent obtained results had the influence of age removed. The reason for the discrepancy between our hypothesis and the findings may be related to the regional cerebral effects of donepezil and the type of tests we used in our study.

As mentioned, there is a tight coupling between working memory and selective attention. The ability to focus our cognitive resources on information relevant to our goals influences working memory performance. Furthermore, attention and working memory are increasingly viewed as overlapping constructs [36]. The working memory types of tasks used in this study require attentional processing and an inability to sustain attention (vigilance) as well as distractibility may cause impairments in these working memory tests. As mentioned previously, attention functioning may be captured exogenously and characterized by "bottom-up" processing or attention may be internally driven and voluntarily allocated in a "top-down" manner. The tests of attention used in this study for the most part, are "top-down" or intentional attention as opposed to "bottom-up" or reactive attention. Gazzaley and Nobre [36] reviewed recent evidence from human neurophysiological studies which demonstrate that top-down modulation serves as a common neural mechanism underlying working memory and that these two cognitive operations engage prefrontal and parietal cortex.

Working memory, as well as attentional vigilance, appears to depend on frontal-parietal network. In addition, donepezil and rivastigmine are also known to possess regional differences in cerebral blood flow. Whereas patients who respond to donepezil exhibit increased cerebral blood flow to the anterior frontal lobe and the parietal lobe [37], patients who respond to rivastigmine exhibit increased blood flow to hippocampal structures and the prefrontal cortex [38]. These findings suggest that donepezil increases regional cerebral blood in those regions of the brain associated with vigilant attentional processing and working memory. These collective findings could potentially explain why donepezil was associated with better performance on the tests of working memory-attention used in our study. Future research might want to focus on measuring the differential effects of donepezil and rivastigmine on more specific factors of attentional processing.

Our findings might have important clinical implications for the treatment of patient's with Lewy Body Dementia (LBD). There have been a few published reports comparing the effectiveness of donepezil versus rivastigmine in treating patients with LBD. Some have reported significant improvement in both cognitive and behavioral measures following treatment with either donepezil or rivastigmine [39], although donepezil was associated with a greater reduction in the total score from the Neuropsychiatric Inventory. A thorough review of the literature by Simard and van Reekum [40] indicated that both donepezil and rivastigmine are efficacious in treating patients with LBD. However, other researchers have reported greater improvement in a range of cognitive and behavioral measures following treatment with rivastigmine as compared to donepezil, in patients with $\mathrm{AD}$ who also had symptoms suggestive of $\mathrm{LBD}$ [41]. A central feature of Lewy body dementia is the presence of disturbances in attention functioning. Hence, given our current findings, the possibility exists that donepezil would be more efficacious in treating the attention-related deficits associated with LBD. Unfortunately, to date there have been no published reports that have compared donepezil and rivastigmine in specifically treating attention deficits in patients with LBD. Future research should be conducted to investigate this possibility.

Although our findings indicate differential effects on attention functioning between donepezil and rivastigmine, there are important limitations that should be considered. Our groups were heterogeneous in regard to diagnosis, with some patients being diagnosed only with $\mathrm{AD}$ but other patients having a mixed diagnosis of $\mathrm{AD}$ and VD. The effect of including patients with dual diagnoses is not known, but this may have affected the findings. However, we attempted to control for this variable by including equal numbers of patient with $\mathrm{AD}$ and with both $\mathrm{AD}$ and $\mathrm{VD}$ within our groups. Hence, no one group contained more patients with VD than any other group. Perhaps the most serious limitation is the range of dosages of donepezil and rivastigmine that were included. Higher dosages of either medication may have a more profound effect on attention and working memory. Review of the data indicates that half of the donepezil group were taking a large $10 \mathrm{mg}$ dosage but only three of the rivastigmine group were taking a larger $9.5 \mathrm{mg}$ dosage. This may explain why rivastigmine was not associated with the greatest benefit in attention and working memory. Further research will need to be conducted to determine if different dosages of these medications result in different impacts on attention and working memory.

\section{Acknowledgement}

This study was not associated with any outside funding. There are no conflicts of interest to report.

\section{References}

1. Ellis JR, Ellis KA, Bartholomeusz CF, Harrison BJ, Wesnes KA, et al. (2006) Muscarinic and nicotinic receptors synergistically modulate working memory and attention in humans. Int J Neuropsychopharmacol 9: 175-189.

2. Levy JA, Parasuraman R, Greenwood PM, Dukoff R, Sunderland T (2000) Acetylcholine affects the spatial scale of attention: evidence from Alzheimer's disease. Neuropsychology 14: 288-298.

3. Broks P, Preston GC, Traub M, Poppleton P, Ward C, et al. (1988) Modelling dementia: effects of scopolamine on memory and attention. Neuropsychologia 26: 685-700.

4. Furey ML, Pietrini P, Haxby JV, Drevets WC (2008) Selective effects of cholinergic modulation on task performance during selective attention. Neuropsychopharmacology 33: 913-923.

5. Davis KL, Mohs RC, Marin D, Purohit DP, Perl DP, et al. (1999) Cholinergic markers in elderly patients with early signs of Alzheimer disease. JAMA 281: 1401-1406.

6. Perry EK, Perry RH, Blessed G, Tomlinson BE (1978) Changes in brain cholinesterases in senile dementia of Alzheimer type. Neuropathol Appl Neurobiol 4: 273-277. 
Citation: Foster PS, Drago V, Roosa KM, Campbell RW, Witt JC, et al. (2016) Donepezil Versus Rivastigmine in Patients with Alzheimer's Disease: Attention and Working Memory. J Alzheimers Neurodegener Dis 2: 002.

7. Coleman AE, Geula C, Price BH, Mesulam MM (1992) Differential lamina distribution of acetylcholinesterase and butyrylcholinesterase containing tangles in the cerebral cortex of Alzheimer's disease. Brain Res 596: 340-344.

8. Geula C, Mesulam M (1989) Special properties of cholinesterases in the cerebral cortex of Alzheimer's disease. Brain Res 498: 185-189.

9. Herholz K, Bauer B, Wienhard K, Kracht L, Mielke R, et al. (2000) In-vivo measurements of regional acetylcholine esterase activity in degenerative dementia: Comparison with blood flow and glucose metabolism. J Neural Transm (Vienna) 107: 1457-1468.

10. McGuinness B, Barrett SL, Craig D, Lawson J, Passmore AP (2010) Attention deficits in Alzheimer's disease and vascular dementia. J Neurol Neurosurg Psychiatry 81: 157-159.

11. Gorus E, De Raedt R, Lambert M, Lemper JC, Mets T (2006) Attentional processes discriminate between patients with mild Alzheimer's disease and cognitively healthy elderly. Int Psychogeriatr 18: 539-549.

12. Perry RJ, Hodges JR (1999) Attention and executive deficits in Alzheimer's disease. A critical review. Brain 122: 383-404.

13. Pignatti R, Rabuffetti M, Imbornone E, Mantovani F, Alberoni M, et al. (2005) Specific impairments of selective attention in mild Alzheimer's disease. J Clin Exp Neuropsychol 27: 436-448.

14. Kadir A, Darreh-Shori T, Almkvist O, Wall A, Grut M, et al. (2008) PET imaging of the in vivo brain acetylcholinesterase activity and nicotine binding in galantamine-treated patients with AD. Neurobiol Aging 29: 1204-1217.

15. Bohnen NI, Kaufer DI, Hendrickson R, Ivanco LS, Lopresti B, et al. (2005) Cognitive correlates of alterations in acetylcholinesterase in Alzheimer's disease. Neurosci Lett 380: 127-132.

16. Klinkenberg I, Sambeth A, Blokland A (2011) Acetylcholine and attention Behav Brain Res 221: 430-442.

17. Kaasinen V, Någren K, Järvenpää T, Roivainen A, Yu M, et al. (2002) Regional effects of donepezil and rivastigmine on cortical acetylcholinesterase activity in Alzheimer's disease. J Clin Psychopharmacol 22: 615-620.

18. Salloway S, Ferris S, Kluger A, Goldman R, Griesing T, et al. (2004) Efficacy of donepezil in mild cognitive impairment: a randomized placebo-controlled trial. Neurology 63: 651-657.

19. Ginani GE, Tufik S, Bueno OF, Pradella-Hallinan M, Rusted J, et al. (2011) Acute effects of donepezil in healthy young adults underline the fractionation of executive functioning. J Psychopharmacol 25: 1508-1516.

20. Foldi NS, White RE, Schaefer LA (2005) Detecting effects of donepezil on visual selective attention using signal detection parameters in Alzheimer's disease. Int J Geriatr Psychiatry 20: 485-488.

21. Bohnen NI, Kaufer DI, Hendrickson R, Ivanco LS, Lopresti BJ, et al. (2005b) Degree of inhibition of cortical acetylcholinesterase activity and cognitive effects by donepezil treatment in Alzheimer's disease. J Neurol Neurosurg Psychiatry 76: 315-319.

22. Schmitt FA, Saxton JA, Xu Y, McRae T, Sun Y, et al. (2009) A brief instrument to assess treatment response in the patient with advanced Alzheimer disease. Alzheimer Dis Assoc Disord 23: 377-383.

23. Kasuya M, Meguro K, Okamura N, Funaki Y, Ishikawa H, et al. (2012) Greater responsiveness to donepezil in Alzheimer patients with higher levels of acetylcholinesterase based on attention task scores and a donepezil PET study. Alzheimer Dis Assoc Disord 26: 113-118.

24. Articus K, Baier M, Tracik F, Kühn F, Preuss UW, et al. (2011) A 24-week, multicentre, open evaluation of the clinical effectiveness of the rivastigmine patch in patients with probable Alzheimer's disease. Int J Clin Pract 65: 790796

25. Gauthier S, Juby A, Rehel B, Schecter R (2007) EXACT: rivastigmine improves the high prevalence of attention deficits and mood and behaviour symptoms in Alzheimer's disease. Int J Clin Pract 61: 886-895.
26. O'Brien KK, Saxby BK, Ballard CG, Grace J, Harrington F, et al. (2003) Regulation of attention and response to therapy in dementia by butyrylcholinesterase. Pharmacogenetics 13: 231-239.

27. Almkvist O, Darreh-Shori T, Stefanova E, Spiegel R, Nordberg A (2004) Preserved cognitive function after 12 months of treatment with rivastigmine in mild Alzheimer's disease in comparison with untreated $\mathrm{AD}$ and $\mathrm{MCl}$ patients. Eur J Neurol 11: 253-261

28. Darreh-Shori T, Almkvist O, Guan ZZ, Garlind A, Strandberg B, et al. (2002) Sustained cholinesterase inhibition in $A D$ patients receiving rivastigmine for 12 months. Neurology 59: 563-572.

29. Hansen RA, Gartlehner G, Webb AP, Morgan LC, Moore CG, et al. (2008) Efficacy and safety of donepezil, galantamine, and rivastigmine for the treatment of Alzheimer's disease: a systematic review and meta-analysis. Clin Interv Aging 3: 211-225

30. Takeda A, Loveman E, Clegg A, Kirby J, Picot J, et al. (2005) A systematic review of the clinical effectiveness of donepezil, rivastigmine and galantamine on cognition, quality of life and adverse events in Alzheimer's disease. Int $\mathrm{J}$ Geriatr Psychiatry 21: 17-28.

31. Caffarra P, Vezzadini G, Copelli S, Dieci F, Messa G, et al. (2007) Comparing treatment effects in a clinical sample of patients with probable Alzheimer's disease treated with two different cholinesterase inhibitors. Acta Biomed 78: 16-21.

32. Matsuzono K, Sato K, Kono S, Hishikawa N, Ohta Y, et al. (2015) Clinical benefits of rivastigmine in the real world dementia clinics of the Okayama Rivastigmine Study (ORS). J Alzheimers Dis 48: 757-763.

33. Scharre DW, Vekeman F, Lefebvre P, Mody-Patel N, Kahler KH, et al. (2010) Use of antipsychotic drugs in patients with Alzheimer's disease treated with rivastigmine versus donepezil: A retrospective, parallel-cohort, hypothesis-generating study. Drugs Aging 27: 903-913.

34. Mesulam M, Guillozet A, Shaw P, Quinn B (2002) Widely spread butyrylcholinesterase can hydrolyze acetylcholine in the normal and Alzheimer brain. Neurobiol Dis 9: 88-93.

35. Giacobini E, Spiegel R, Enz A, Veroff AE, Cutler NR (2002) Inhibition of acetyl- and butyryl-cholinesterase in the cerebrospinal fluid of patients with Alzheimer's disease by rivastigmine: correlation with cognitive benefit. J Neural Transm (Vienna) 109: 1053-1065.

36. Gazzaley A, Nobre AC (2012) Top-down modulation: bridging selective attention and working memory. Trends Cogn Sci 16: 129-135.

37. Yoshida T, Ha-Kawa S, Yoshimura M, Nobuhara K, Kinoshita T, et al. (2007) Effectiveness of treatment with donepezil hydrochloride and changes in regional cerebral blood flow in patients with Alzheimer's disease. Ann Nucl Med 21: $257-265$

38. Potkin SG, Anand R, Fleming K, Alva G, Keator D, et al. (2001) Brain metabolic and clinical effects of rivastigmine in Alzheimer's disease. Int J Neuropsychopharmacol 4: 223-230.

39. Bhasin M, Rowan E, Edwards K, McKeith I (2007) Cholinesterase inhibitors in dementia with Lewy bodies: a comparative analysis. Int J Geriatr Psychiatry 22: 890-895.

40. Simard M, van Reekum R (2004) The acetylcholinesterase inhibitors for treatment of cognitive and behavioral symptoms in dementia with Lewy bodies. $J$ Neuropsychiatry Clin Neurosci 16: 409-425.

41. Touchon J, Bergman H, Bullock R, Rapatz G, Nagel J, et al. (2006) Response to rivastigmine or donepezil in Alzheimer's patients with symptoms suggestive of concomitant Lewy body pathology. Curr Med Res Opin 22: 49-59. 